\title{
Demo Abstract: Automated Diagnostics for AHU-VAV Systems Using Pattern Matching
}

\author{
Adam Regnier \\ Drexel University \\ Department of Civil, Architectural, \\ and Environmental Engineering \\ Philadelphia, PA \\ ar626@drexel.edu
}

\author{
Jin Wen, $\mathrm{PhD}$ \\ Drexel University \\ Department of Civil, Architectural, \\ and Environmental Engineering \\ Philadelphia, PA \\ jw325@drexel.edu
}

\author{
Jesse Schwakoff \\ Drexel University \\ Department of Civil, Architectural, \\ and Environmental Engineering \\ Philadelphia, PA \\ jjs339@drexel.edu
}

\begin{abstract}
Faults in commercial HVAC systems can result in energy waste of up to $30 \%$ of the total usage. This demonstration exhibits a novel application for automated fault detection and diagnosis (AFDD) for air handling units (AHUs) and variable air volume units (VAVs) in building HVAC systems. The application has been designed to facilitate low-cost implementation and fault monitoring, while maintaining high diagnostic accuracy and low false alarm rates. This is accomplished via the use of unsupervised machine learning methods and analytic redundancies, combining pattern-matching methods, principal component analysis (PCA) methods, and Bayesian network analysis into a single AFDD application. The benefit of using these automated methods is the ability to adapt to the built up custom systems found with AHUVAV installations, thereby minimizing the engineering effort required for implementation.
\end{abstract}

\section{Introduction}

Studies have shown that the occurrence and undetected presence of faults within commercial HVAC systems can increase the energy consumption of these systems by as much as $30 \%$. Additionally, these faults often negatively impact occupant comfort, indoor air quality, and maintenance/service costs. These negative impacts can be eliminated with the adoption of an effective automated fault detection and diagnosis strategy, yet no widely adopted AFDD strategies for air handling units (AHUs) currently exist.

This lack of commercial adoption is a result of a variety of technical and market-driven hurdles that need to be overcome, including:

- Systems are built-up (customized) differently for each application
- Sensors are typically of low quality and sparsely distributed

- Operation is continuously transient and non-linear, and transitions through multiple operational modes

- Existing strategies typically have unacceptably high false alarm rates, and dont provide adequate information for building operators to take intelligent action

Herein, a novel AFDD application is demonstrated that was specifically designed to address the aforementioned issues. It has been connected to monitor two buildings in Philadelphia, and the demonstration consists of showing the process for setting up the application, the ongoing output from real-time monitoring, as well as the results from experiments when faults were artificially injected into these buildings.

\section{Technology Overview}

The program being demonstrated has a number of key features that result in accurate and usable diagnostic outputs to a building operator while maintaining a low implementation cost. Initial set-up of the application only requires that the data points be mapped from the building management system to the application, and then the application can immediately start to learn the system characteristics.

The AFDD process consists of two distinct phases: retrocommissioning of the building to get it operating properly and then ongoing monitoring for faults. This application is designed to assist an engineer with the former and to perform the latter in a fully automated manner once the building is operating in a satisfactory manner.

Every AHU-VAV system is unique, with a wide range of sizes, sensor configurations, components, and control strategies existing in different buildings. As a result, utilizing an application that is automatically trained and adaptable to a variety of different system types is essential to minimize the upfront engineering costs. Additionally, the application demonstrated here has been designed to fully utilize the advantages of systems that are heavily instrumented with sensors but also to maintain high accuracy in systems that contain only minimal sensor instrumentation. By testing this application on a variety of different building and system types, the flexibility has been demonstrated to be effective for nearly all AHU-VAV system/control configurations. 


\section{Methods}

The first step in implementing the AFDD package is to identify pre-existing faults in the system. This can be performed via a standard commissioning process or can be performed using the Bayesian network fault engine included in this application. During the initial connection process, the application learns the system characteristics and identifies issues to address before going online.

Once the building is deemed to be operating in a satisfactory manner the online AFDD process then commences. This process uses a pattern matching algorithm to compare the current system operation with the learned historical operation, and identifies historical fault-free conditions that are similar to the current operational conditions.

If similar historical operational conditions are identified, these data are used to generate a fault-free PCA model. The current data are then compared to the historical PCA model using squared prediction error (SPE) to determine if the data are faulty or fault free. However, if no similar historical operational data are identified, the new data are passed directly to the commissioning Bayesian network for validation. If the data are found to be fault-free, the process is halted and no further action is taken, but if the data are found to be faulty then the diagnostic algorithm is triggered.

The first step of the diagnostic process is to use a PCA reconstruction method to determine which measurement values are responsible for causing the fault to be flagged. This information is then passed to a Bayesian network fault engine using ternary logic to indicate if each measurement is lower, normal, or higher than expected. The diagnostic Bayesian network combines this information with firstprinciple based rules and generates a probabilistic output indicating what fault is most likely responsible for the abnormal system operation, and the confidence in the diagnosis.

\section{Results}

At the time of writing, this application has been tested and demonstrated at three commercial buildings of various sizes, with a wide variety of HVAC systems and control strategies. The testing has included investigation of the implementation and computational requirements, the efficacy of fault detection and diagnosis, and the ability to generate usable data for a building operator.

\subsection{Implementation Requirements}

One advantage of this method mentioned above, beyond the efficacy of fault detection, is the low cost of implementation and monitoring. The cost of implementation consists firstly of connecting/mapping the points from the BMS to the AFDD application. Secondly, there is a manual review required at the outset to ensure that there are not existing faults that need to be addressed prior to putting the AFDD program online. This process is aided by the Bayesian network fault engine, but does require some human interaction to differentiate between system faults and unique system idiosyncrasies.

In terms of data storage, this application requires the ability to store a maximum of 6 weeks of data to adequately encapsulate the full spectrum of operational conditions. This equates to approximately $0.005 \mathrm{~GB}$ for each $\mathrm{AHU}$ and 0.002
GB for each VAV box. This data can be acquired at the outset if available or during the system operation as necessary. The computational speed running the application through Matlab on an Intel i7-3770 PC averages 2.9 seconds per system component (AHU or VAV-box). The application is presently configured to run every 30 minutes, and using this timing allows for the capability to presently monitor over 600 components in near-real-time.

\subsection{Diagnostic Results}

To date, this application has been tested in three different buildings across the full spectrum of seasonal and operational conditions. The testing has consisted of identifying faults naturally occurring in these buildings as well as extensive experimental testing using faults that are artificially injected via control overrides or physical manipulation of the system components.

Overall, this application was found to accurately detect $93 \%$ of all faults, with a false alarm rate of $2.9 \%$. The faults that were not detected were all of very low severity, with negligible impact on system operation, and all faults with a significant comfort, safety, or energy impact were accurately detected. The false alarm rate exceeds the targeted ceiling of $1 \%$, but this is expected to improve with further updates to the application that will improve the programs robustness to false alarms.

\subsection{Diagnostic Output}

The output of a commercially-viable AFDD application should be easily interpreted by users with a variety of experience and expertise with HVAC systems. To accommodate a wide variety of users, this application provides a simple, prioritized output of all faults, but also allows the user to delve into a detailed explanation of why a fault was flagged and to view the raw data that generated the fault notification. This allows a building operator to access as much, or as little information as desired, and to pass detailed information to a service technician. Each fault diagnosis is also accompanied by information about the comfort, safety, and energy impacts of the fault to assist with prioritization and decision-making.

\section{Discussion and Future Work}

This application has been demonstrated to be an effective, low-cost solution for a wide variety of HVAC systems that cover most typical AHU-VAV configurations. However, some further refinement may be necessary to ensure robust operation in less common system types that have not yet been investigated. Going forward, testing and demonstration of this application is in the process of being extended to additional buildings for this purpose, as well as to generate additional results data.

\section{Acknowledgments}

Our thanks to the US Department of Energys Consortium for Building Energy Innovation (CBEI) for support and funding of this research. Additionally, key technical support for the experimental testing was provided by United Technologies Research Center (UTRC), and support for testing, data archiving, and Volttron integration was provided by Pacific Northwest National Laboratory (PNNL). 